\title{
Reduction in Cutis Verticis Gyrata after Transsphenoidal Pituitary Resection of Growth Hormone-Secreting Adenoma
}

\author{
Daniel Pinggera ${ }^{1}$ Delia Anna Marsoner ${ }^{1} \quad$ Marcel Seiz-Rosenhagen $^{2} \quad$ Claudius Thomé $^{1}$ \\ Christian Franz Freyschlag ${ }^{1}$
}

${ }^{1}$ Department of Neurosurgery, Medical University Innsbruck, Innsbruck, Austria

${ }^{2}$ Department of Neurosurgery, University Medical Center Mannheim, University of Heidelberg, Mannheim, Germany

Address for correspondence Christian Franz Freyschlag, MD, Department of Neurosurgery, Medical University Innsbruck, Anichstrasse 35, 6020 Innsbruck, Austria (e-mail: christian.freyschlag@tirol-kliniken.at).

Indian J Neurosurg 2016;5:84-88.

\begin{abstract}
Aim Cutis verticis gyrata (CVG) describes a hypertrophy and folding of the skin that appears as gyration of the scalp. Secondary CVG is a common symptom of growth hormone $(\mathrm{GH})$-producing adenoma of the pituitary and an early sign of acromegaly. We hypothesized that surgical removal of a pituitary adenoma with a consecutive decrease in hormone levels will cause a reduction in gyration.

Methods Imaging and laboratory examination of 10 patients (median age: 44 years) with $\mathrm{GH}$-producing adenoma have been evaluated retrospectively. Surgical treatment consisted of endonasal transsphenoidal resection of the adenoma, followed by endocrinologic and radiographic follow-up. CVG was calculated as area under the curve of the scalp on preoperative coronal T1-weighted MRI, compared with postoperative follow-up imaging after 12 months (AUC, measured in $\mathrm{cm}^{2}$ ). In laboratory examination, the levels of insulin-like growth factor-1 (IGF-1) were analyzed accordingly as its levels are constant throughout the day.

Results After 12 months, we found a statistically significant $(p=0.008)$ correlation

Keywords

- acromegaly

- cutis verticis gyrate

- pituitary adenoma between the decrease in IGF-1 levels (median: $219.00 \mathrm{ng} / \mathrm{mL}$ ) and the reduction in CVG (median: $0.12 \mathrm{~cm}^{2}$ ).

Conclusion Resection of $\mathrm{GH}$-producing pituitary adenoma leads to a decrease in CVG in patients suffering from acromegaly, which is strongly related to the levels of IGF-1
\end{abstract}

\section{Introduction}

Pituitary adenomas account for 10 to $20 \%$ of all intracranial tumors and growth hormone (GH)-producing adenoma may occur in up to $10 \%$ of this population. ${ }^{1} \mathrm{GH}$-producing adenomas cause an aberrant secretion of $\mathrm{GH}$ and, consecutively, insulinlike growth factor-1 (IGF-1), leading to the distinctive features of acromegaly. ${ }^{2}$ The prevalence of acromegaly is $60 / 1,000,000$ with an incidence of 3 to 4 per million per year. ${ }^{3}$

Acromegaly may present with a variety of different symptoms, ranging from subtle signs of acral overgrowth, jaw prognathism, fasting hyperglycemia, and hyperhidrosis to more severe diseases such as diabetes mellitus, hypertension, and cardiac failure. Besides medical history, physical findings, and imaging, diagnosis is made by measurement of high-serum IGF-I and high-glucosesuppressed GH levels. ${ }^{4}$ Transsphenoidal surgery is the treatment of choice according to current guidelines, whereas control rates are strongly dependent on the neurosurgeons experience and the size of the adenoma. ${ }^{5,6}$ Therapeutic success is defined by normalization of IGF- 1 and received

December 26, 2015

accepted

February 3, 2016

published online

July 19, 2016
DOI http://dx.doi.org/

10.1055/s-0036-1584591. ISSN 2277-954X. (c) 2016 Neurological Surgeons' Society
of India

License terms

(®) $\ominus \circledast$ 
GH serum levels $(<1 \mu \mathrm{g} / \mathrm{L}$ and a GH nadir of $<0.4 \mu \mathrm{g} / \mathrm{L}$ in oral glucose tolerance test [OGTT]). ${ }^{2,7}$

Skin changes have been recognized an important component of acromegaly. IGF-1 is stimulated by $\mathrm{GH}$ and produced primarily by the liver. It activates the IGF-1 receptor, a tyrosine kinase receptor, located in the cell membrane. There are no major differences in relation to sex or circadian rhythm. IGF-1 receptor signaling regulates skin development, growth and differentiation of fibroblasts, and keratinocytes. ${ }^{8}$ This fact leads to severe cutaneous manifestations such as cutis verticis gyrata (CVG), acanthosis nigricans, or hyperhidrosis.

In our study we wanted to focus on CVG, a disorder characterized by thickening of the scalp due to hypertrophy of the skin, leading to skin folds and grooves that resemble the gyri of the brain surface. It has an estimated prevalence of 1 in 100,000 in males and 0.026 in 100,000 in females. ${ }^{9}$ The term was first used in 1901 by Unna. ${ }^{10}$ It may be classified into a primary and a secondary form. Primary CVG has no obvious cause, usually appears after puberty, and is often associated with neurologic conditions, such as epilepsy or mental retardation. ${ }^{11}$ Secondary CVG is frequently associated with endocrine or neoplastic conditions such as acromegaly, myxedema, or amyloidosis. ${ }^{8,12}$ The number of folds varies from 2 to 12, and the direction is mostly anterior to posterior. They cannot be corrected by pressure or traction and the skin color is normally unaffected.

These skin changes are well known to impair the quality of life in patients with acromegaly. 8,13 Therefore, we want to investigate whether transsphenoidal surgery of GH-producing adenoma leads to a decrease in CVG in patients suffering from acromegaly related to the levels of IGF-1.

\section{Materials and Methods}

Data of all patients with surgically treated GH-producing adenoma and the signs of acromegaly between 2006 and 2012 were retrospectively evaluated for presence of CVG. As institutional review board approval is not needed for retrospective cohorts in Austria, informed consent was not required. We identified 12 patients with CVG who underwent endonasal transsphenoidal surgery for GHproducing adenoma; 2 patients had to be excluded due to a lack of imaging data or serum levels of IGF-1, resulting in 10 eligible patients. Serum levels of IGF-1 were recorded using identical IGH arrays (standard value: $56-201 \mathrm{ng} / \mathrm{mL}$ ). Magnetic resonance imaging (MRI) was performed preoperatively and 12 months after surgery.

Defined areas under the curve (AUC) of preoperative coronal T1-weighted MRI were compared with postoperative follow-up imaging after 12 months (AUC, measured in $\mathrm{cm}^{2}$ ). A standardized measurement protocol was applied to mess CVG (-Fig. 1). The red and blue are were measured together to minimize errors in measurement. All measurement were done pre- and postoperatively with Agfa HealthCare IMPAX 6 (Agfa HealthCare GmbH, Bonn, Germany).

All statistical analyses were conducted using IBM SPSS Statistics 21 (IBM Corp., Armonk, New York, United States). Differences with an error probability of less than 0.05 were

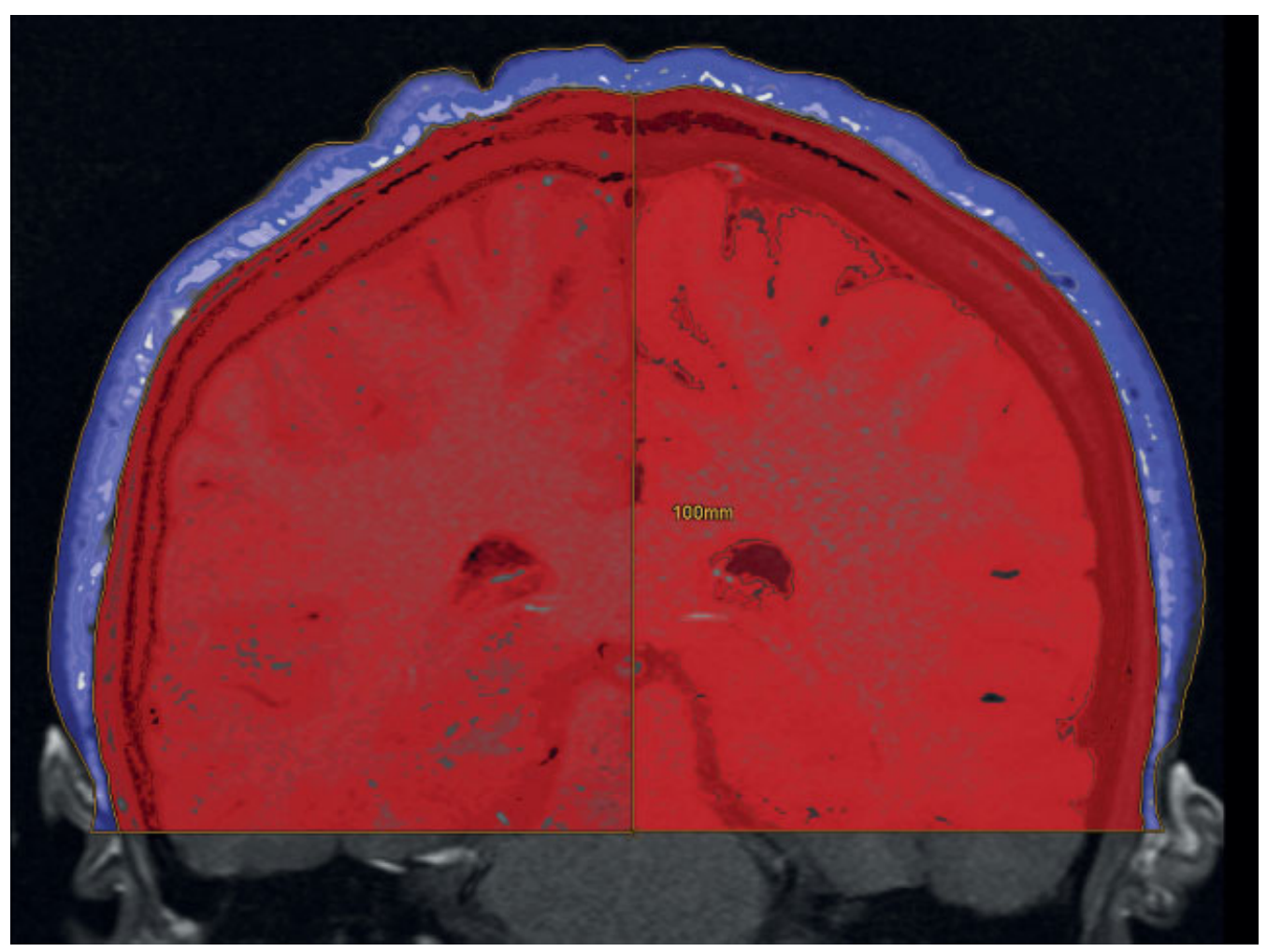

Fig. 1 Illustration of standardized measurement protocol ${ }^{1}$ : The coronal slice of T1-weighted MRI depicting the internal auditory canal bilaterally is identified. ${ }^{2} \mathrm{~A} 100$-mm-long line is drawn from the skull periosteum caudally in the midline. ${ }^{3}$ A second horizontal line is drawn perpendicularly to reach the skin laterally. ${ }^{4}$ The surface of the long skull and the skin are followed to measure the area under the curve (red and blue area). 


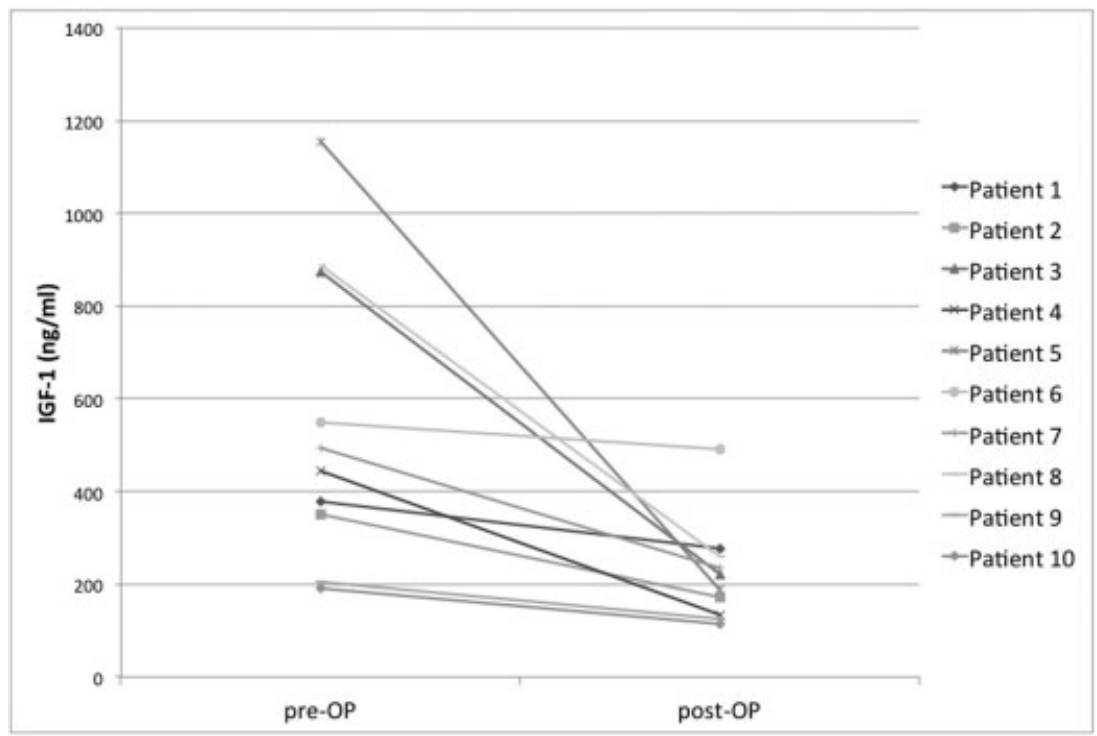

Fig. 2 Change in AUC total $\left(\mathrm{cm}^{2}\right)$. AUC, area under the curve.

considered statistically significant. A $t$-test was used to evaluate the reduction in the AUC related to the IGF-1 serum levels as well as to determinate the difference between the AUC pre- and postoperatively.

\section{Results}

In 10 patients we found a $4: 6$ male/female ratio with a mean age at surgery of 44 years (range: $31-74$ years). The mean total AUC (red and blue area together, Fig. 1) at surgery was $13.72 \mathrm{~cm}^{2}$ (range: $12.82-14.66 \mathrm{~cm}^{2}$ ) and $13.64 \mathrm{~cm}^{2}$ (range: $12.75-14.34 \mathrm{~cm}^{2}$ ) 12 months after surgery. In nine cases a decrease in the AUC was seen, with a slight increase in one patient (-Fig. 2). This patient was afterward assigned to further drug therapy. The mean reduction in the AUC was $0.12 \mathrm{~cm}^{2}$ (range: $-0.8-0.52 \mathrm{~cm}^{2}$ ).
The mean IGF-1 levels at time of surgery was $469 \mathrm{ng} / \mathrm{mL}$ (range: 190-1,155) and $205 \mathrm{ng} / \mathrm{mL}$ (range: 114-490) at the 12-month follow-up evaluation. In all 10 cases the serum levels of IGF-1 decreased (-Fig. 3). We were able to demonstrate that both the decreases in the total AUC correlate to the decreasing serum levels of IGF-1 according to the $t$-test $(p=0.008)$.

\section{Discussion}

To the best of our knowledge, this is the first study dealing with changes in CVG related to postoperative reduction in $\mathrm{GH}$ levels. It could be demonstrated that a postoperative reduction in IGF1 will lead to a reduction in CVG.

Although the diagnosis of CVG is primarily clinical, imaging plays a role in differentiating primary CVG from

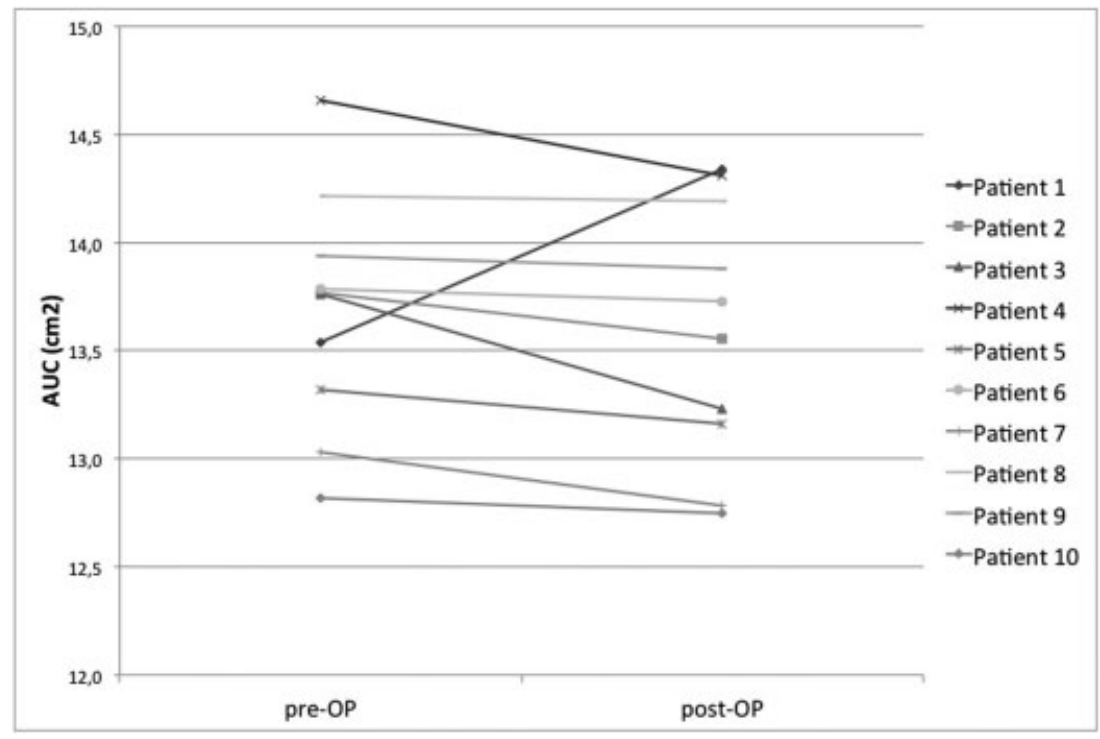

Fig. 3 Change in IGF-1 levels (ng/mL). IGF-1, insulin-like growth factor-1. 
secondary CVG. The appearance of CVG on computed tomography (CT) and MRI is characteristic, and because of the anterior-posterior direction of the folds, the coronal plane is regarded best to visualize CVG. ${ }^{14-16}$ In four of our patients, imaging data were available only of a pituitary MRI protocol, including coronal plane only around sella, but CVG was clearly visible. Contrary to the available literature, we only reviewed cases of secondary CVG alone, leading to a small sample size.

Despite the small number of patients, our epidemiologic data are in agreement with the literature. The mean patient age was over 40 years with a female predominance., 16,17

Similar to the study by Schöfl et al, we only evaluated the levels of IGF-1, as the GH assay used at our center changed during the observed period, and in light of the enormous interassay variability, data could be found misleading. ${ }^{17}$ Furthermore, recent data confirm discordance between $\mathrm{GH}$ and IGF-1 after surgery, so that we decided to focus on one marker. ${ }^{18}$ The increase in total CVG in one patient cannot be explained definitively, since levels of IGF-1 were decreasing, but the AUC of the skin was decreasing too. Overall, the correlation was better seen in total AUC. We hypothesize that the measurement is more accurate when done completely.

All patients showed postoperative normalized levels of IGF-1, and we believe that this decrease in IGF-1 stops and reverses the hypertrophy of the skin, as described for other cutaneous manifestations as acanthosis nigricans and oily skin. ${ }^{8,19,20}$ This may contribute a better health-related quality of life as skin manifestations are often complained by patients; nevertheless, the normal levels of IGF- 1 alone improve health-related quality of life. ${ }^{8,12}$ Reduction in CVG is probably only a side effect of surgically treated acromegaly, but perhaps this could be a decision-making aid to undergo surgery, considering the "cosmetic" disturbances mentioned by patients. The limitations of our study include its retrospective nature and its limited followup of 1 year.

\section{Conclusion}

Transsphenoidal resection of GH-producing adenoma of the pituitary leads to a decrease in CVG in patients suffering from acromegaly related to the decreasing levels of IGF-1.

\section{Disclosure}

The authors report no conflict of interest concerning the materials or methods used in this study or the findings specified in this paper.

\section{Congress}

Portions of this work were presented in poster and oral form at the EANS Annual Meeting 2013, Tel Aviv, Israel, and at the 49th Annual Meeting of the Austrian Neurosurgical Society 2013, Innsbruck, Austria.
Funding

None.

Conflicts of Interest

All the authors certify that they have no affiliations with or involvement in any organization or entity with any financial interest (such as honoraria; educational grants; participation in speakers' bureaus; membership, employment, consultancies, stock ownership, or other equity interest; and expert testimony or patent-licensing arrangements), or nonfinancial interest (such as personal or professional relationships, affiliations, knowledge, or beliefs) in the subject matter or materials discussed in this manuscript.

\section{Acknowledgments}

None.

\section{References}

1 Ostrom QT, Gittleman H, Farah P, et al. CBTRUS statistical report: primary brain and central nervous system tumors diagnosed in the United States in 2006-2010. Neuro-oncol 2013;15(Suppl 2): ii1-ii56

2 Melmed S. Medical progress: acromegaly. N Engl J Med 2006; 355(24):2558-2573

3 Holdaway IM, Rajasoorya C. Epidemiology of acromegaly. Pituitary 1999;2(1):29-41

4 Katznelson L. AACE acromegaly guidelines. Endocr Pract 2011;17: Suppl 4

5 Melmed S, Colao A, Barkan A, et al; Acromegaly Consensus Group. Guidelines for acromegaly management: an update. J Clin Endocrinol Metab 2009;94(5):1509-1517

6 Nomikos P, Buchfelder M, Fahlbusch R. The outcome of surgery in 668 patients with acromegaly using current criteria of biochemical “cure.". Eur J Endocrinol 2005;152(3): 379-387

7 Holdaway IM, Bolland MJ, Gamble GD. A meta-analysis of the effect of lowering serum levels of GH and IGF-I on mortality in acromegaly. Eur J Endocrinol 2008;159(2):89-95

8 Centurión SA, Schwartz RA. Cutaneous signs of acromegaly. Int J Dermatol 2002;41(10):631-634

9 El-Husseiny MYJ, Moiemen N. Primary cutis gyrata: review of literature and a successful new surgical approach. Eur J Plast Surg 2010;33(3):153-157

10 Unna P. Cutis gyrata cutis. Monatschr Prakt Derm 1907;45:227-233

11 Chang GY. Cutis verticis gyrata, underrecognized neurocutaneous syndrome. Neurology 1996;47(2):573-575

12 Yang JJ, Sano DT, Martins SR, Tebcherani AJ, Sanchez AP. Primary essential cutis verticis gyrata-case report. An Bras Dermatol 2014;89(2):326-328

13 Guitelman M, Abreu A, Espinosa-de-los-Monteros AL, Mercado M. Patient-focussed outcomes in acromegaly. Pituitary 2014;17 (Suppl 1):S18-S23

14 Kolawole TM, Al Orainy IA, Patel PJ, Fathuddin S. Cutis verticis gyrata: its computed tomographic demonstration in acromegaly. Eur J Radiol 1998;27(2):145-148

15 Alorainy IA. Magnetic resonance imaging of cutis verticis gyrata. J Comput Assist Tomogr 2008;32(1):119-123

16 Okamoto K, Ito J, Tokiguchi S, Ishikawa K, Furusawa T, Sakai K. MRI in essential primary cutis verticis gyrata. Neuroradiology 2001;43(10):841-844 
88 Reduction in CVG Pinggera et al.

17 Schöfl C, Franz H, Grussendorf M, et al; participants of the German Acromegaly Register. Long-term outcome in patients with acromegaly: analysis of 1344 patients from the German Acromegaly Register. Eur J Endocrinol 2013;168(1): 39-47

18 Zeinalizadeh M, Habibi Z, Fernandez-Miranda JC, Gardner PA, Hodak SP, Challinor SM. Discordance between growth hormone and insulin-like growth factor-1 after pituitary surgery for acromegaly: a stepwise approach and management. Pituitary 2015;18(1):48-59

19 Dahbar M, Danilowicz K, Malavela M, et al. Cutaneous manifestations of acromegaly. Dermatol Argent 2009;15:186-190

20 Ben-Shlomo A, Melmed S. Skin manifestations in acromegaly. Clin Dermatol 2006;24(4):256-259 